\title{
Fractional Charge of Quarks and Fractal Properties of Hadrons
}

\author{
A. Bhattacharya ${ }^{1 *}$, S. Pal $^{2}$, D. S. Bhattacharya ${ }^{3}$ and P. Dhara ${ }^{1}$ \\ ${ }^{1}$ Department of Physics, Adamas University, Kolkata, India \\ ${ }^{2}$ Department of Physics, Basanti Devi College, Kolkata,700029 India \\ ${ }^{3}$ Julius-Maximilians University of Würzburg, Germany \\ Email: aparajita.bhattacharay@adamasuniversity.ac.in
}

\begin{abstract}
The origin of fractional charge of a quark is investigated considering the fractal structure of a hadron. Hadron is suggested to be a fractal object with fractal dimension 9/2. Describing quark as a quasiparticle in an analogy with quasi particle in Fractional Quantum Hall Effect, the filling factors are extracted which show large fractional plateau. It is suggested that quarks behave like quasi particles and the fractional charges of quarks can be attributed to the fractal behavior of a hadron.
\end{abstract}

Keywords: fractal dimension, hadron, fractional quantum hall effect (FQHE), fractional charges, quark.

\section{Introduction}

The origin of fractional charge of quarks and quasi particles is one of the most important topics of research for a long time. The most well confirmed example of fractional charges of quasi particles are studied in the context of Fractional Quantum Hall Effect (FQHE) [1]. This effect results from strong magnetic field giving rise to fractionally charged quasi particles which carry currents. Chung [2] has investigated the fractional charges in the context of integer-fraction principle of digital electronic charge which relates the confinement and collectivity of fractional charges. There are a large number of efforts to confirm the fractional charge experimentally but till now the results are null [3-6]. This is also interpreted as the permanent confinement of quarks within the hadrons. Fractional charges of quasiparticles are studied by Heiblum et al. [7] in the context of FQHE - liquid. They have suggested the charge as $\mathrm{e}^{*}=\mathrm{e} / 3$ with filling factor $\nu=1 / 3$. Patton et al. [8] have investigated the fractional charge of impurity bound state in FQHE by exact diagonalisation of electron Green's function. They have predicted that the fractional charge is consistent with Laughlin [9] quasi particles where charges are given by $\mathrm{e}^{*}=0.31 \mathrm{e}$ for $\nu=1 / 3$ and $\mathrm{e}^{*}=0.25 \mathrm{e}$ for $\nu=2 / 5$. Zielke et al. [10] have calculated the co-tunnelling current for electrons that tunnel between two quantum edges via a quantum dot and for the quasiparticles with fractional charges e/4 and e/2 which tunnel through anti dot.

The dimensionality of a quantum system is a very important property which governs the dynamics of the system itself. The electrons behave differently which includes Luttinger liquid in one (1D) and the Fractional Quantum Hall Effect in two (2D) dimension. Three dimensional (3D) FQHE has been studied by Halperin [11] theoretically which is recently observed experimentally by Tang et al. [12] in the metal - insulator transition $Z_{r T e}$. It is interesting to investigate the behavior of the quasi particles in fractal dimension. The concept of fractal dimensions are now widely used to understand the properties and dynamics of chaos, multi particle production, inflationary universe, information dimension and many more [13-14]. The dimensionality and fractional charges of quasi particles are studied by a number of authors. In the current work we have studied the fractional charges of quarks considering the fractal dimension of hadrons. In our previous works we have studied the hadron properties describing it as a fractal object with fractal dimension 9/2 [15-17]. We have suggested that each quark in presence of the chromo-magnetic vacuum behaves like a quasi particle in an analogy with the quasi particles in Fractional Quantum Hall Effect and their fractional charges can be attributed to the fractal dimension of the hadrons. 


\section{Formulation}

The properties of a system depends strongly on the dimension of the system. For last few decades a statistical model for hadron has been suggested which is widely used to study the properties of hadrons. In this model a quark is supposed to be surrounded by a virtual sea of $q \bar{q}$ pair where the valence quark only determine the quantum number of quark constituting the hadron. The most important prediction of the statistical model is the prediction of fractal dimension of a hadron. The effective number density of quarks are related to the square of the wave function and can be expressed as:

$$
\psi(r)=A\left(r_{0}-r\right)^{3 / 4} \theta(r-r)
$$

The wave function has a remarkable feature that it is non analytic in nature and shows scaling property. The fractal dimension for hadron has been found to be $\mathrm{D}=9 / 2$ [16] when the topological dimension $D_{T}$ $=3$. The fractal dimension indicates the measure of complexity of a system which can not be shown by the integer dimensions. We have suggested that a quark behaves like a quasi particle in QCD vacuum and can be represented by the wave function (1). The quasi particle wave function has been investigated extensively in the context of FQHE. Laughlin [9] has suggested an elegant wavefunction of the ground and the excited states of this new state of matter composed of dressed electrons considering the electron interaction and explains the energy gap in FQHE which experiences the fractional interaction. The ground state is separated by a gap $\Delta$ which is described by a unique wave function. The many body variational trial collective wave function at a filling factor $\nu=1 / \mathrm{m}$ can be presented by taking the power of polynomial that describes the filled Landau level and suggested to have the form:

$$
\phi_{m}=\left(z-z_{0}\right)^{2 p} \exp \left(-1 / 4|z|^{2}\right)
$$

where $\phi$ describes $\mathrm{N}$ identical particles with charge $\mathrm{Q}=2$ p. Jain [18] has pointed out that the fractional filling factor can be explained by a Composite Fermion, which is a quasi particle described by transformation of properties of particles as a collective effect of attachment of flux quanta. The redefinition of the wave function is required for describing a quasi particle. It is well known that the coulomb interaction gives rise to a new set of energy gap occurring at a new filling factor. The gap is replaced by a new gap with a filling factor

$$
\nu=\frac{\nu^{*}}{2 p \nu^{*}+1}
$$

For FQHE the ground state properties of these unique phases where the low excitations above ground states are fractionally charged and the effective charge of the quasi particle with added extra magnetic flux is given by:

$$
e^{*}=\frac{\nu^{*}}{2 p \nu^{*}+1}
$$

The equation (1) describes the wave function for a quark derived in the context of the statistical model. We suggest that the quarks in the virtual sea of $q \bar{q}$ pairs behave like quasi particles in the presence of chromo magnetic vacuum. The exponent of the wave function $3 / 4$ is related to the Landau filling factor as described in FQHE with $2 \mathrm{p}=3 / 4$. For any given value of charge, $\nu^{*}$ can be obtained which gives us the values of quasi particle filling factor $\nu^{*}$ using equation (4). With input of the charge of $\mathbf{u}$ quark (Q $=2 / 3)$ in (4), we have obtained $\nu^{*}=4 / 3$ with $2 \mathrm{p}=3 / 4$ from (1) whereas the charge of $\mathbf{d}$ quark $(\mathrm{Q}=$ $-1 / 3)$ quark yields $\nu^{*}=-4 / 15$. So we find that a filling factor $\nu^{*}=4 / 3$ will generate a charge of the quasiparticle $2 / 3$ whereas $\nu^{*}=-4 / 15$ generates charge of quasiparticle $-1 / 3$. The filling factor is defined as electron to magnetic flux ratio in the context of FQHE. In the present investigation the exponent of the statistical model wave function (1) for a quark is used to find the filling factor of fractional charges of $\mathbf{u}$ and $\mathbf{d}$ quarks. The fractal dimension of hadron is suggested to be related to the fractional filling factor of the quasi particles described by the wave function (1).

\section{Conclusion}

In the current work we have investigated the fundamental question of the origin of fractional charges of the quarks. We have suggested that the quark behave as quasi particles in the presence of the QCD vacuum 
which possesses chromo-magnetic character. Statistical model wave function has an unique property of describing fractal properties of quarks. We have used it as a trial wave function for quasi particles. It has been suggested that the quasiparticle wave function is subjected to the fractional statistics [19]. Cruz [20] has suggested that the fractal geometry of nature is manifested in the FQHE and the study of fractal structure is important for deeper understanding of FQHE and the quantum path whereas Pal [13] has argued that FQHE must be characterized by the fractal dimension and suggested a wave function which describes a Laughlin type anyons for Housdorff dimension between 1 and 2. Yarlagadda [22] has derived a wave function at a filling factor $\nu=n /(2 \mathrm{~m}+1)$ where the wavefunction is $(2 \mathrm{~m}+1)^{\text {th }}$ power of exact wave function for the $\mathrm{n}^{\text {th }}$ filled Landau level. Jacak [21] has investigated fractional charges of quarks from the holographic principle. Kempkes et al [14] have constructed an electron wave function in fractal dimensions. In the current work the filling factor is extracted from the exponent of the wave function in an analogy or suggestion of Laughlin wave function describing the quasi particle in the context of FQHE. The wave function has remarkable feature that it is non analytic in nature and possesses scaling properties. It is observed that filling factor $4 / 3$ reproduces the charge of 'u' quark whereas filling factor $-4 / 15$ reproduces the charge of 'd' quark. They represent the odd denominator plateau. For filling factor $4 / 15(<1)$, the lowest Landau level spin up is partially occupied whereas for the filling factor $4 / 3(>$ 1), lowest Landau lavel with spin up is filled and the lowest Landau level with spin down is partially occupied. It may be mentioned that initially there is appearance of the $1 / 3$ plateau but with the concept of the composite Fermion, the fractional plateaus with larger values are confirmed with most of them having odd denominator. The understanding of FQHE with the fractal dimension of the quasi particles are very interesting area and important for the understanding of the properties and dynamics of the quantum paths. We have estimated the filling factors with the input of charge of the quarks along with the input of the exponent of our trial wave function. The filling factor shows large fractional plateau indicating the quasi particle behavior. It may be suggested that the fractal dimension of quarks is related to the quasi particles behavior which may also be in turn responsible for the quark confinement.

\section{References}

1. D. C. Tsui, H. L. Stormer, and A. C. Gossard, Phys. Rev. Lett. 48(22), 1559 (1982)

2. D. -Yu -Chung, J. Mod. Phys. 7, 1150 (2016)

3. V. J. Goldman and B. Su, Science 267 (5200), 1010 (1995)

4. J.D.F. Franklin, I. Zailer, C.J.B. Ford, P.J. Simpson, J.E.F. Frost, D.A. Ritchie, M.Y. Simmons and M. Pepper, Surf. Sci 361, 17 (1996)

5. Eun-Ah Kim, M. J. Lawler, S. Vishveshwara and E. Fradkin, Phys. Rev B 74, 155324 (2006)

6. J. H. Wang et al., J. Inst 7, (2012)

7. M. Heiblum, Adv. in Solid State Physics 40, pp:21-34, (ASSP-40) Book Series.

8. K. R. Patton and M. R. Geller, New. J. Phys. 16, 023004 (2014)

9. R. B. Laughlin, Phys. Rev. Lett. 50, 1395 (1983)

10. R. Zielke, B. Braunecker and D. Loss, Phys. Rev. B 86, 235307 (2012).

11. B. I. Halperin, Phys. Rev. Lett. 52, 1583 (1984)

12. F. Tang, Y. Ren, P. Wang, R. Zhong, J. Schneeloch, et al., Nature 569, 537 (2019)

13. S. Manna, B. Pal, W. Wang, and A. E. B. Nielsen, arXiv:1907.03193v1(Cond-matt-str-el), (2019)

14. S. N. Kempkes, M.R. Slot, S. E. Freeney, S. J. M. Zevenhuizen, D. Vanmaekelbergh et al., Nat. Phys. 15(2), $127(2019)$

15. S. N. Banerjee, A. Bhattacharya, B. Chakrabarti and S. Banerjee, Int. J. Mod. Phys. A 16(2), 201 (2001)

16. S. N. Banerjee, A. Bhattacharya, B. Chakrabarti and S. Banerjee, Int. J. Mod. Phys. A 17(32), 4939 (2002)

17. R. Saha, R. Ghosh, D. Bhattacharya, and A. Bhattacharya, AIP Conf. Proce. 2072, 020013 (2019)

18. J. Jain, Phys. Rev. Lett. 63, 199 (1989)

19. F. Wilczek, Phys. Rev. Lett. 49, 957 (1982)

20. W. da. Cruz, arXiv:Cond-mat/0304398v6], (2004).

21. J. E. Jacak, arXiv: 1704.06560, [Physics.gen-ph] (2017)

22. S. Yarlagadda, Physica B 223, 640 (1996) 